\title{
Decreased Pulmonary Function Measured in Children Exposed to High Environmental Relative Moldiness Index Homes
}

\author{
Stephen J. Vesper ${ }^{*}$, , Larry Wymer $^{1}$, Suzanne Kennedy ${ }^{2}$ and L. Faye Grimsley ${ }^{3}$ \\ ${ }^{1}$ United States Environmental Protection Agency, Cincinnati, Ohio, 45268, USA \\ ${ }^{2}$ Rho Federal Systems Division, Inc., Chapel Hill, North Carolina, 27517, USA \\ ${ }^{3}$ School of Public Health and Tropical Medicine, Tulane University, New Orleans, Louisiana, 70112, USA
}

\begin{abstract}
Background: Exposures to water-damaged homes/buildings has been linked to deficits in respiratory health. However, accurately quantifying this linkage has been difficult because of the methods used to assess water damage and respiratory health.
\end{abstract}

\begin{abstract}
Purpose: The goal of this analysis was to determine the correlation between the water-damage, as defined by the Environmental Relative Moldiness Index (ERMI) value in an asthmatic child's home, and the child's pulmonary function measured by spirometry, "forced expiratory volume in one second, percent predicted" or FEV1\%.
\end{abstract}

Methods: This analysis utilized data obtained from the "Heads-off Environmental Asthma in Louisiana" (HEAL) study. The children ( $\mathrm{n}=109), 6$ to 12 years of age, who had completed at least one spirometry evaluation and a dust sample collected for ERMI analysis from the home at approximately the same time as the spirometry testing, were included in the analysis. Statistical evaluation of the correlation between ERMI values and FEV1\% was performed using the Spearman's Rank Correlation analysis. The relationship between ERMI values and FEV1\% was performed using B-spline regression.

Results: The average ERMI value in the HEAL study homes was 7.3. For homes with ERMI values between 2.5 and 15 , there was a significant inverse correlation with the child's lung function or FEV1\% measurement (Spearman's rho - 0.23 ; $\mathrm{p}=0.03$ ), i.e. as the ERMI value increased, the FEV1\% value decreased.

Conclusions: Measures of water-damage (the ERMI) and clinical assessments of lung function (FEV1\%) provided a quantitative assessment of the impact of water-damaged home exposures on children's respiratory health.

Keywords: FEV1\%, ERMI, lung function, children.

\section{INTRODUCTION}

Exposure to water-damaged, moldy homes/buildings has been linked to respiratory disease [1, 2], including asthma [3, 4]. Many methods have been used to estimate the water-damage and/or mold contamination in homes/buildings but probably a visual and/or olfactory inspection is the most common. This is not highly quantitative. Similarly, many diverse methods have been used to estimate the respiratory health impact from exposures to water-damaged environments including, caregiver diaries, healthcare utilization records, phone surveys, etc. [5]. These are not clinically precise. In this study, a standard metric for quantifying waterdamage/mold contamination in homes, the Environmental Relative Moldiness Index (ERMI), and a clinically-based spirometry test called FEV1\% or "forced expiratory volume in 1 second, percent predicted" were utilized to quantitatively relate exposures to lung function.

*Address correspondence to this author at the United States Environmental Protection Agency, 26 West M. L. King Drive, Cincinnati, OH 45268, USA; Tel: +1 513569 7367; E-mail: vesper.stephen@epa.gov
The US Environmental Protection Agency (US EPA) in conjunction with the Department of Housing and Urban Development (HUD) created a standardized assessment to quantify water-damage and mold contamination in US homes called the ERMI [6]. For the ERMI analysis, a DNAbased technology, mold specific quantitative polymerase chain reaction, was used to measure the concentration of 36 indicator molds in settled-dust samples. The concentration of each of the 36 molds is mathematically treated to produce a single number, the ERMI value for that home $[6,7]$. The ERMI scale ranges from -10 to 20 for most homes but a few homes have ERMI values up to about 40 . For convenience of use, the ERMI scale is divided into quartiles. About $50 \%$ of homes in the US have low ERMI values of -10 to 0 . Those in the third quartile range from 0 to 5 and the highest quartile is above 5 [6].

Clinical testing with spirometry of a child's lung function provides a standardized evaluation of a child's respiratory health. Measurement of lung function by spirometry is reportedly both objective and reproducible [8]. The goal of this analysis was to determine the correlation between the water-damage, i.e. ERMI value, in an asthmatic child's home 
and the child's pulmonary function, as measured by FEV1\%. This analysis utilized data obtained from the "Heads-off Environmental Asthma in Louisiana" (HEAL) study [9-12] but was not part of the original HEAL study design. The HEAL study concerned the impact of the 29 August 2005 hurricane Katrina on the children in the affected homes in New Orleans, LA.

\section{MATERIALS AND METHODOLOGY}

The HEAL study was approved by the National Institute of Environmental Health Sciences and the Tulane and Louisiana State Universities' Institutional Review Boards. Many previous publications have describe the HEAL study, the methods used, the population demographics, the exposures measured, and the outcomes [9-12]. No attempt will be made to repeat this background but it is briefly summarized below.

Hurricane Katrina impacted the city of New Orleans in August of 2005 and recruitment for the HEAL study began in February 2007 and was completed by March 2008 [9]. Children $(\mathrm{n}=182)$ were recruited from New Orleans schools and pre-screened by telephone. They were eligible if they were 4-12 years old and a parent or guardian reported they had asthma; lived in Orleans Parish or neighboring parishes that were affected by the hurricane Katrina and subsequent flooding; and met criteria for moderate-to-severe asthma at the time of the baseline clinical evaluation, as described earlier [9]. At the time of the study, about $60 \%$ of homes had completed some level of renovation/remediation, in $26 \%$ of homes efforts were on-going and in $13 \%$ work had not started [11].

Many of the participants were not necessarily receiving appropriate care for their asthma. As a result of their enrollment, each participant received excellent standard of care and therapy for their asthma [12]. At the time of the baseline clinical visit spirometry testing was completed using a EasyOne Spirometer (ndd Medical, Inc., Andover, $\mathrm{MA}$ ) and the calculation of FEV $1 \%$ was completed following American Thoracic Society protocol (https://www. thoracic.org/statements/resources/pfet/PFT2.pdf). Shortly after the clinical evaluation, a home visit was made for dust collection and other environmental evaluations [9].

Of the children enrolled in HEAL, 67\% were African American, and $25 \%$ came from households with annual incomes $<\$ 15 \mathrm{~K}$ and $51 \%$ making $<30 \mathrm{~K}$ [12]. Therefore the participants were generally of low socioeconomic status. HEAL children were symptomatic, averaging 6.6 symptom days in the 2 weeks before the baseline sampling, and had frequent unscheduled visits to clinics or emergency departments $(76 \%$ had at least one unscheduled visit in the preceding 3 months) [9].

This current analysis only included the 109 children who were at least 6 years of age and therefore eligible to perform spirometry testing. They had to have completed at least one spirometry evaluation and a dust sample had to have been obtained from the home at approximately the same time as the spirometry testing. For 50 of the children, the spirometry testing and dust collecting and ERMI analysis were performed twice during this 12 month study (repeats at least 6 months apart).
Methods and MSQPCR assays have been reported previously for performing the ERMI analysis [13]. Briefly, the standard reaction assays contained $12.5 \mu \mathrm{l}$ of "Universal Master Mix" (Applied Biosystems Inc., Foster City, CA), 1 $\mu \mathrm{l}$ of a mixture of forward and reverse primers at $25 \mu \mathrm{M}$ each, $2.5 \mu \mathrm{l}$ of a $400 \mathrm{nM}$ TaqMan probe (Applied Biosystems Inc.), $2.5 \mu \mathrm{l}$ of $2 \mathrm{mg} / \mathrm{ml}$ fraction $\mathrm{V}$ bovine serum albumin (Sigma Chemical, St. Louis, MO) and $2.5 \mu 1$ of DNA free water (Cepheid, Sunnyvale, CA). To this mix was added $5 \mu \mathrm{l}$ of the DNA extract from the sample. All primer and probe sequences used in the assays have been published [14]. Primers and probes were synthesized commercially (Applied Biosystems, Inc.).

Statistical evaluation of the correlation between ERMI values and FEV1\% was performed using the Spearman's Rank Correlation analysis. Outliers in the data were evaluated using the Grubbs test. Among subjects with ERMI and FEV1\% measurements at both time points $(\mathrm{N}=50)$, differences between base line and follow-up were evaluated via the Wilcoxon signed rank test. Neither ERMI nor FEV 1\% were found to differ significantly from baseline to follow-up ( $p=0.96$ and $p=0.89$, respectively). The average values for the ERMI and FEV1\% test results were utilized in the Spearman's Rank Correlation analysis. The relationship between ERMI values and FEV1\% was performed using Bspline regression [15] showing the $95 \%$ confidence interval. The analyses were performed in SAS version 9.3 (SAS Institute, Cary NC) and R version 2.14 (R Foundation for Statistical Computing, Vienna, Austria).

\section{RESULTS}

The average ERMI value for the homes in the HEAL study was 7.3 (standard deviation \pm 4.6) and the average FEV $1 \%$ value was $95 \%$ (standard deviation +15 ). The Grubbs' test identified one ERMI value of 30.61 in a single home as an outlier, compared to the average ERMI of 7.3. Similarly, the FEV1\% levels for two of the children were measured at $38 \%$ and $43 \%$ in their initial clinic visit, compared to $95 \%$ for the average. Therefore these two sets of results were also removed from further analysis, leaving 106 ERMI and FEV1\% data pairs. The paired ERMI and FEV 1\% data is shown in Fig. (1) with the regression line for FEV1\% showing a downward slope as the homes' ERMI values increased.

The possible relationship between the ERMI value of the home and the FEV1\% value for the child's lung function was visualized by plotting a B-spline regression using two interior quantile knots and 95\% confidence intervals (Fig. 2). (We also examined spline curves with one and three knots, but the two knot spline curve provided the best fit to the data.) The first knot or change in curve direction fell in the third quartile of the ERMI scale, which is bounded on the low side by an ERMI value of 0 and on the high side with an ERMI value of 5 [6]. Therefore, the value of 2.5 was selected, precisely in the middle of the third quartile, to divide the homes into two groups; those with ERMI values below 2.5 and those with ERMI values above 2.5.

For the set of homes with ERMI values below 2.5, there was no significant correlation between ERMI values and FEV1\% results (Spearman's rho $-0.1 ; p=0.7$ ). For the set of 


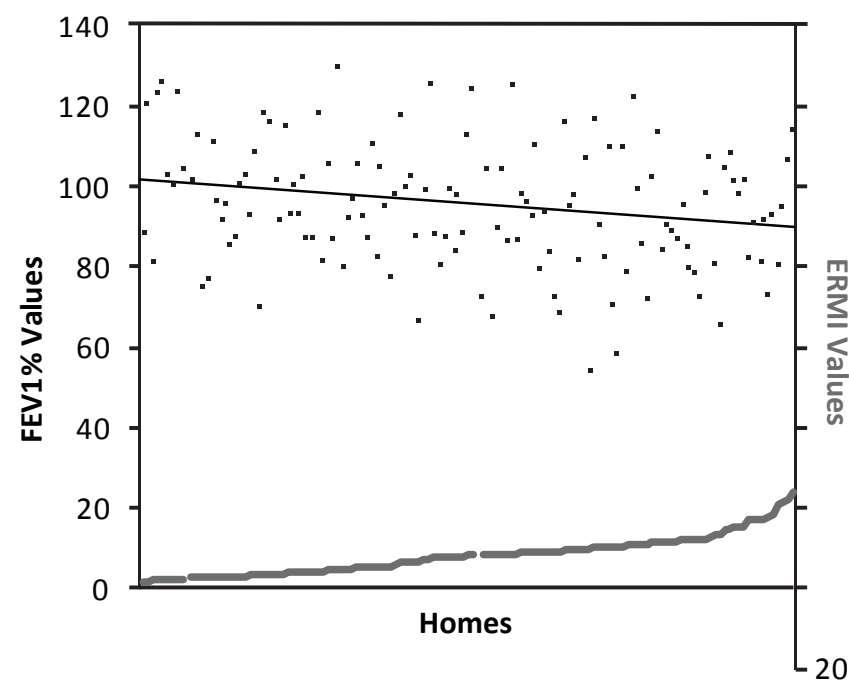

Fig. (1). The paired Environmental Relative Moldiness Index (ERMI) value in each asthmatic child's home (gray squares, z-axis) and the child's "forced expiratory volume in 1 second percentage" (FEV1\%) value (black dots, y-axis). The regression line for FEV1\% shows a downward slope as the homes' ERMI values increased.

homes with ERMI values between 2.5 and 15, there was a significant inverse correlation with the child's lung function or FEV1\% measurement (Spearman's rho -0.23 ; $p=0.03$ ), i.e. as the ERMI value increased, the FEV1\% value decreased. (There were only six homes with ERMI values above 15; too few to evaluate the relationship between ERMI and FEV1\%, as noted by the flattening of the B-spline at about that point). The peak FEV1\% of about $100 \%$ was at an ERMI value of approximately 2.5 . The FEV1\% values declined to a low of about $70 \%$ at an ERMI value of 15 (Fig. 2).

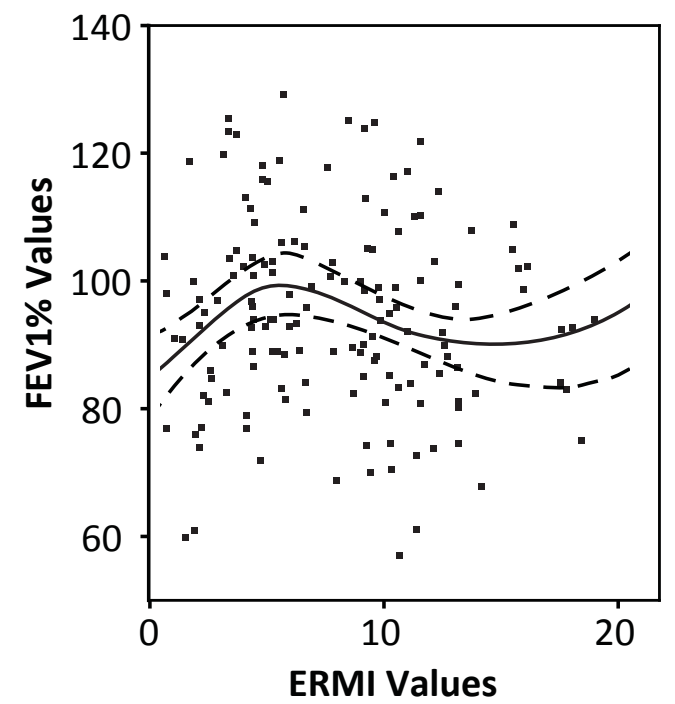

Fig. (2). The association between the Environmental Relative Moldiness Index (ERMI) values and the corresponding "forced expiratory volume in 1 second percentage" (FEV1\%) values were evaluated by plotting a B-spline regression (solid black line) using two interior quantile knots. The $95 \%$ confidence limits for the curve are shown as hatched lines. The peak FEV1\% of about $100 \%$ was at an ERMI value of approximately 2.5 . The FEV1\% values declined to about $70 \%$ at an ERMI value of 15 .

\section{DISCUSSION}

Exposure of a child to a New Orleans home with water damage above 2.5 on the ERMI scale was likely to result in a deficit in the child's normal lung function. Norbäck et al. [16] similarly demonstrated that the lung function of adults declined over time when exposed to damp homes. Of course, there are many chemical and biological exposures in waterdamaged homes. Therefore, no specific cause(s) for the respiratory deficit can be assigned. Rather this analysis adds to the growing scientific literature that indicates that exposure to water-damaged homes should be reduced [2].

Reponen et al. [3, 4] conducted a prospective study of asthma development from infancy to age seven and the physician-based diagnosis of asthma. They found that infants exposed to homes with ERMI values above 5 were much more likely to develop asthma. In a study of three widely dispersed cities in the US (Boston, Kansas City, and San Diego), the homes of the children with asthma had average ERMI values of 8.7 [17]. This is very similar to the average ERMI value of 7.3 found in the water-damaged homes of the asthmatic children in New Orleans.

We acknowledge that this analysis has many limitations. Since this analysis was not specifically a part of the original study plan, we were limited to a relative small sample size in which the environmental sampling and clinical testing coincided. In addition, we did not have large enough data set for a multivariate analysis. However, previous results from the HEAL study showed exposures to allergens of mice (Mus m 1), dust mites (Der p 1) and cockroach (Bla g 1) in only $60 \%, 35 \%$ and $20 \%$ of homes respectively and at low mean concentrations [11]. These exposures do not appear to be significant confounding exposures but of course we have not measured all possible exposures including indoor air pollution, pesticides, etc. In spite of these limitations, our results do suggest, but do not prove, that there is a quantitative relationship between exposures to waterdamaged homes and the exposed child's respiratory function.

It does not take a hurricane to create water-damaged homes. In fact, each year millions of homes suffer waterdamage from obvious sources like flooding and "hidden" problems like the dripping pipe in the wall. These waterdamaged homes may be contributing to the asthma epidemic [18] which costs the US billions of dollars each year [19]. Since the costs continue to escalate, methods to prevent asthma development or exacerbation are needed. One important step might be remediating water-damaged homes. Combining more quantitative measures of water-damage like the ERMI and clinical assessments of the child's health through spirometry might provide the motivation to prevent children from being exposed to water-damaged homes.

\section{CONFLICT OF INTEREST}

The U.S. Environmental Protection Agency (EPA) through its Office of Research and Development, collaborated in the research described here. Although this work was reviewed by EPA and approved for publication it may not necessarily reflect official EPA policy. Mention of trade names or commercial products does not constitute endorsement or recommendation by the EPA for use. Since 
MSQPCR technology is patented by the US EPA, the Agency has a financial interest in its commercial use.

\section{ACKNOWLEDGEMENTS}

We thank Katherine Loizos for preparation of the figures.

\section{Funding}

The HEAL study was funded, in whole or in part, with federal funds from the National Institute of Environmental Health Sciences (NIEHS), National Institutes of Health (NIH), under contract NO1-ES-55553. Additional funding support for HEAL was provided by the Merck Childhood Asthma Network and the National Center on Minority Health and Health Disparities under the auspices of the Foundation for the NIH. Other organizations that contributed to HEAL include the National Toxicology Program (NIEHS), the U.S. Environmental Protection Agency (Cincinnati, $\mathrm{OH}$ ), and the de Laski Family Foundation. The Clinical and Translational Research Center of Tulane and Louisiana State Universities Schools of Medicine was supported in whole or in part by funds provided through the Louisiana Board of Regents RC/EEP.

\section{REFERENCES}

[1] IOM (Institute of Medicine, National Academies of Science.) Damp indoor spaces and health. Washington, D.C: The National Academies Press 2004.

[2] WHO (World Health Organization) Europe. WHO guidelines for indoor air quality: dampness and mould. Copenhagen, Denmark: WHO 2009.

[3] Reponen T, Vesper S, Levin L, et al. High environmental relative moldiness index during infancy as a predictor of age seven asthma. Ann Allergy Asthma Immun 2011; 107: 120-6.

[4] Reponen T, Lockey J, Berstein DI, et al. Infants exposed to specific molds correlated with age seven asthma. J Allergy Clin Immun 2012; 130: 639-644.

[5] Bime C, Nguyen J, Wise RA. Measures of asthma control. Curr Opin Pulm Med 2012; 18: 48-56.

[6] Vesper SJ, McKinstry C, Haugland RA, et al. Development of an environmental relative moldiness index for homes in the U.S. J Occup Environ Med 2007; 49: 987-90.
[7] Meheust D, Gangneux J-P, Reponen T, Wymer L, Vesper S, Le Cann P. Correlation between Environmental Relative Moldiness Index (ERMI) values in French dwellings and other measures of fungal contamination. Sci Total Environ 2012; 438: 319-24.

[8] Tepper RS, Wise RS, Covar R, et al. Asthma outcomes: pulmonary physiology. J Allergy Clin Immunol 2012; 129(3 Suppl): S65-87.

[9] Chulada PC, Kennedy S, Mvula MM, et al. The Head-off Environmental Asthma in Louisiana (HEAL) study - Design, methods and population. Environ Health Perspect 2012; 120: 15929.

[10] Grimsley LF, Wildfire J, Lichtveld M, et al. Few associations found between mold and other allergen concentrations in the home versus skin sensitivity from children with asthma after hurricane Katrina in the Head-Off Environmental Asthma in Louisiana study. Int J Pediatr 2012:427358. doi: 10.1155/2012/427358.

[11] Grimsley LF, Chulada PC, Kennedy S, et al. Indoor environmental exposures for children with asthma enrolled in the HEAL study, post-Katrina New Orleans. Environ Health Perspect 2012; 120: 1600-6.

[12] Mitchell H, Cohn RD, Wildfire J, et al. Implementation of evidence-based asthma interventions in post-Katrina New Orleans: the Head-off Environmental Asthma in Louisiana (HEAL) study. Environ Health Perspect 2012; 120: 1607-12.

[13] Haugland RA, Varma M, Wymer LJ, Vesper SJ. Quantitative PCR of selected Aspergillus, Penicillium and Paecilomyces species. Syst Appl Microbiol 2004; 27: 198-210.

[14] Haugland RA, Vesper SJ. Identification and quantification of specific fungi and bacteria. United States patent US 6387652. 2002 May.

[15] de Boor C. A practical guide to splines. New York: Springer Verlag 1978.

[16] Norbäck D, Zock JP, Plana E, et al. Lung function decline in relation to mould and dampness in the home: the longitudinal European Community Respiratory Health Survey ECRHS II. Thorax 2011; 66: 396-401.

[17] Vesper S, Barnes C, Ciaccio CE, et al. Higher Environmental Relative Moldiness Index (ERMI) values measured homes of asthmatic children in Boston, Kansas City and San Diego. J Asthma 2013; 50: 155-61.

[18] CDC (Centers for Disease Control and Prevention): Trends in Asthma Prevalence, Health Care Use, and Mortality in the United States, 2001-2010. Avaialble from: http://www.cdc.gov/nchs/data/d atabriefs/db94.pdf [Accessed July 2012].

[19] Mudarri D, Fisk WJ. Public health and economic impact of dampness and mold. Indoor Air 2007; 17: 226-35.

Received: August 15, 2013

Revised: November 1, 2013

Accepted: November 26, 2013

(C) Vesper et al.; Licensee Bentham Open.

This is an open access article licensed under the terms of the Creative Commons Attribution Non-Commercial License (http://creativecommons.org/licenses/by-nc/3.0/) which permits unrestricted, non-commercial use, distribution and reproduction in any medium, provided the work is properly cited. 